\title{
Performance Analysis in Cellular NETWORKS CONSIDERING THE QOS BY RETRIAL QUEUEING MODEL WITH THE FRACTIONAL GUARD Channels Policies
}

\author{
Dang ThanhChuong ${ }^{1}$, Hoa Ly Cuong ${ }^{1}$, \\ Pham Trung Duc ${ }^{1}$ and Duong Duc Hung ${ }^{2}$ \\ ${ }^{1}$ Faculty of Information Technology, University of Sciences, Hue University, Viet Nam \\ ${ }^{2}$ Hue University, Viet Nam
}

\begin{abstract}
In this article, a retrial queueing model will be considered with persevering customers for wireless cellular networks which can be frequently applied in the Fractional Guard Channel (FGC) policies, including Limited FGC (LFGC), Uniform FGC (UFGC), Limited Average FGC (LAFGC) and Quasi Uniform FGC $(Q U F G C)$. In this model, the examination on the retrial phenomena permits the analyses of important effectiveness measures pertained to the standard of services undergone by users with the probability that a fresh call first arrives the system and find all busy channels at the time, the probability that a fresh call arrives the system from the orbit and find all busy channels at the time and the probability that a handover call arrives the system and find all busy channels at the time. Comparison between four types of the FGC policy can befound to evaluate the performance of the system.
\end{abstract}

\section{KEYWORDS}

Cellular Mobile Networks, Retrial, FGC, QoS.

\section{INTRODUCTION}

In the present, with the aid of the rapid development of wireless and mobile networks, many queueing models, especially retrial queues, have been proposed to evaluate the actual performances of these network systems. In cellular networks, it is important to design network models that the handover calls are more prior than the fresh calls properly. The concerns are limited resources, for example, a number of channels, sharings, and competitions in a certain cell (the collisions between the handover calls and the fresh calls), which can lead to the call interruptions when a user frequently moves among cells in the network as cell edges. Therefore, it is necessary that the appropriate policy admitting and processing the arrival calls minimize congestion risks of the system, including the handover and fresh calls. The call admission controls have been proposed to fulfillQoS requirements [1].

The research [1] and [2] first introduced the application of the retrial queueing models on evaluating cellular mobile networks involving the fractional guard channel policy to minimize the handover probability. Then [3] constructed the novel model and obtained some important results but that model bases only one probability $\theta$. In later years [4] and [5] reapplied and renovated [3] by considering the additional probability of the fresh calls $\theta^{\prime}$. [6] used the fractional guard channel without retrial customers. [7] improved [3] by using the fractional guard channel, but it considered only one probability $\theta$. The fractional guard channel policy allocates resources in the 
cellular mobile network to reserve for the handover calls. This policy is considered the general form of the guard channel ones. When the system has available resources, the fresh call requirements are accepted by a certain probability, depending on the cell states (a number of the busy channels).

Ramjee [8] first proposed the fractional guard channel policy with the accepted probability of the fresh calls $\beta_{i}\left(0 \leq \beta_{i} \leq 1, i=\overline{0, c}\right.$, where $c$ is the number of the channels), and it depends on the number of the operating channels. The problem is that the optimal probability $\beta_{i}$ is chosen by the fractional guard channel policy. The limited fractional guard channel LFGC, the uniform fractional guard channel UFGC, the limited average fractional guard channel LAFGC and the quasi-uniform fractional guard channel QUFGC policies have been initiated based on the features of different network systems.

To generalize the above results, the research can be examined the retrial queueing model whose form is $M / M / c /(c+L)$ in combination with the fractional guard channel policies and the characteristic parameters, such as the probability that a fresh call first arrives and then it enters the orbit and the impatient customer probability that a call resumes to enter the servers. In contrast, it is unsuccessful and eternally departs and the additional features are the probability $\theta$ $(0<\theta \leq 1)$ that a call first enters the orbit when failing and the probability $\theta^{\prime}\left(0<\theta^{\prime} \leq 1\right)$ which can be considered as a call next enters the orbit when failing, whereas [7] has only one fixed probability $\theta$ for all calls entering the orbit. The main aim of our model is to lessen possibilities of being blocked with retrial and handover calls, significant factors to accomplish QoS, with the additional measure which is a novel attribute in comparison with the aforementioned works.

The organization of the article is as follows, In Section 2, the detailed problems with the parameters and the models are described. The analysis results will be presented evaluated the model performances in Section 3.

\section{ANALYSis Model}

\subsection{Performance Analysis of Fractional Guard Channel}

The model that we use in this study is some what like the models in [3], [4], [5] and [7] by considering the retrial customers for the fresh and handover calls. The salient point is that we implement the fractional guard channel with the two probabilities of the fresh calls (Figure 1). In the guard channel model, the guard channels reserve for the handover calls. It means that a fresh call arrives in the system and is accepted with the probability $\beta_{i}\left(0 \leq \beta_{i} \leq 1, i=\overline{0, c}\right.$, where $c$ is the number of the channels) and the system has at least a vacant channel, otherwise it will be rejected. A handover call is denied when all channels of the system are busy. Also in this model, the fairness is considered in the resource stores for all calls, including the fresh and handover calls. The innovations are that implement the fractional guard channels (Definition 2.1) with the limited capacity of the orbit. 


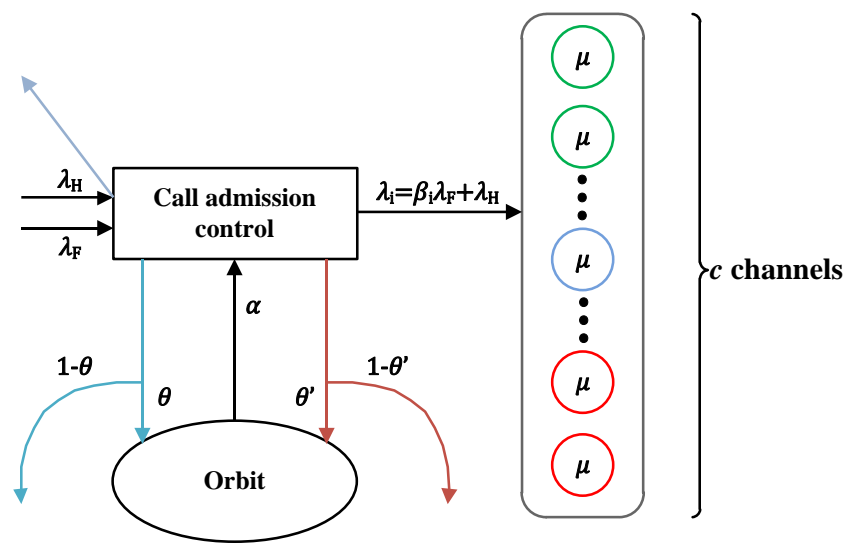

Figure 1.The retrial queueing model in the cellular mobile network.

Definition 2.1 [7]: A fresh or retrial call is admitted by the system in accordance with the fractional guard channel policy. The handover calls are accepted if the system exists a certainly available channel, and the fresh calls only are served with the probabilities $\beta_{i}\left(0 \leq \beta_{i} \leq 1\right)$ depending on the system state.

As the aforementioned issue, we consider the fractional guard channel policies, such as LFGC, UFGC, LAFGC, and QUFGC. In addition, we assume that the system consists of $c$ the operation channels, $g$ the permission channels, the accepted probability of the fresh calls $p(0 \leq p \leq 1)$, and the average value $r(r \in \mathbb{R}, 0 \leq r \leq c)$.

- LFGC: $\beta_{i}=1(0 \leq i \leq g-1), \beta_{g}=p, \beta_{i}=0(g+1 \leq i<c)$.

- LAFGC: $\beta_{i}=1(0 \leq i \leq c-\lfloor r\rfloor-2), \beta_{c-\lfloor r\rfloor-1}=1-r+\lfloor r\rfloor, \beta_{i}=0(c-\lfloor r\rfloor \leq i<c)$.

- UFGC: $\beta_{i}=p(0 \leq i<c)$.

- QUFGC: $\beta_{i}=1(0 \leq i \leq g-1), \beta_{i}=p(g \leq i<c)$.

Obviously, we have $\beta_{c}=0$.

\subsection{The Parameters}

Considering a certain cell in the cellular mobile network, the system is modeled in Figure 1. The arrival calls will be admitted by the call admission control with the fractional guard channel policies. The interarrival times for fresh and handover calls follow the exponential distribution with the rate $\lambda_{F}$ and $\lambda_{H}$, respectively. Let $\lambda$ is defined as the interarrival time between consecutively incoming calls regardless of fresh and handover calls, thus we have $\lambda=\lambda_{F}+\lambda_{H}$. Also, the served time follows the exponential distribution with the rate $\mu$.

A call first arriving the system is blocked due to lacking or allocating the resources. Now it is:

- Permanently depart the system with the probability $1-\theta(0<\theta \leq 1)$.

- or enter the orbit with the probability $\theta$.

In this model, the orbit is considered a queue (capacity $L$ ) to store the blocked calls (or the fresh calls).Similarly, when the retrial calls are unsuccessful, they will:

- Permanently depart the system with the probability $1-\theta^{\prime}\left(0<\theta^{\prime} \leq 1\right)$.

- or continue entering the orbit with the probability $\theta^{\prime}$. 
International Journal of Computer Networks \& Communications (IJCNC) Vol.13, No.4, July 2021

In addition, the retrial rate for the fresh calls from the orbit to the system is $\alpha$.

The fresh or retrial calls enter the system with the probability $\beta_{i}$ in accordance with the state system. A handover call is served immediately if the system exists at least one vacant channel. In contrast, it will permanently depart and does not enter orbit.

\subsection{Modelling}

The system is illustrated by the two-dimensional continuous-time Markov chains $X=$ $\{(I(t), J(t)), t \geq 0\}$, where $I(t)(0 \leq I(t) \leq c)$ are the numbers ofthecustomers being served by the system, and $J(t)(0 \leq J(t) \leq L)$ are the numbers of the customerswaiting to enter the system at the time $t$. The state space of the above chains is depicted by Figure 2 , where $(i, j)$ are the states that the numbers of the occupied channels at the time tarei $(i=0,1,2, \ldots, c-g, c-g+$ $1, \ldots, c)$, and the numbers of the customers in the orbit at the time $\operatorname{tarej}(j=0,1,2, \ldots, L)$.

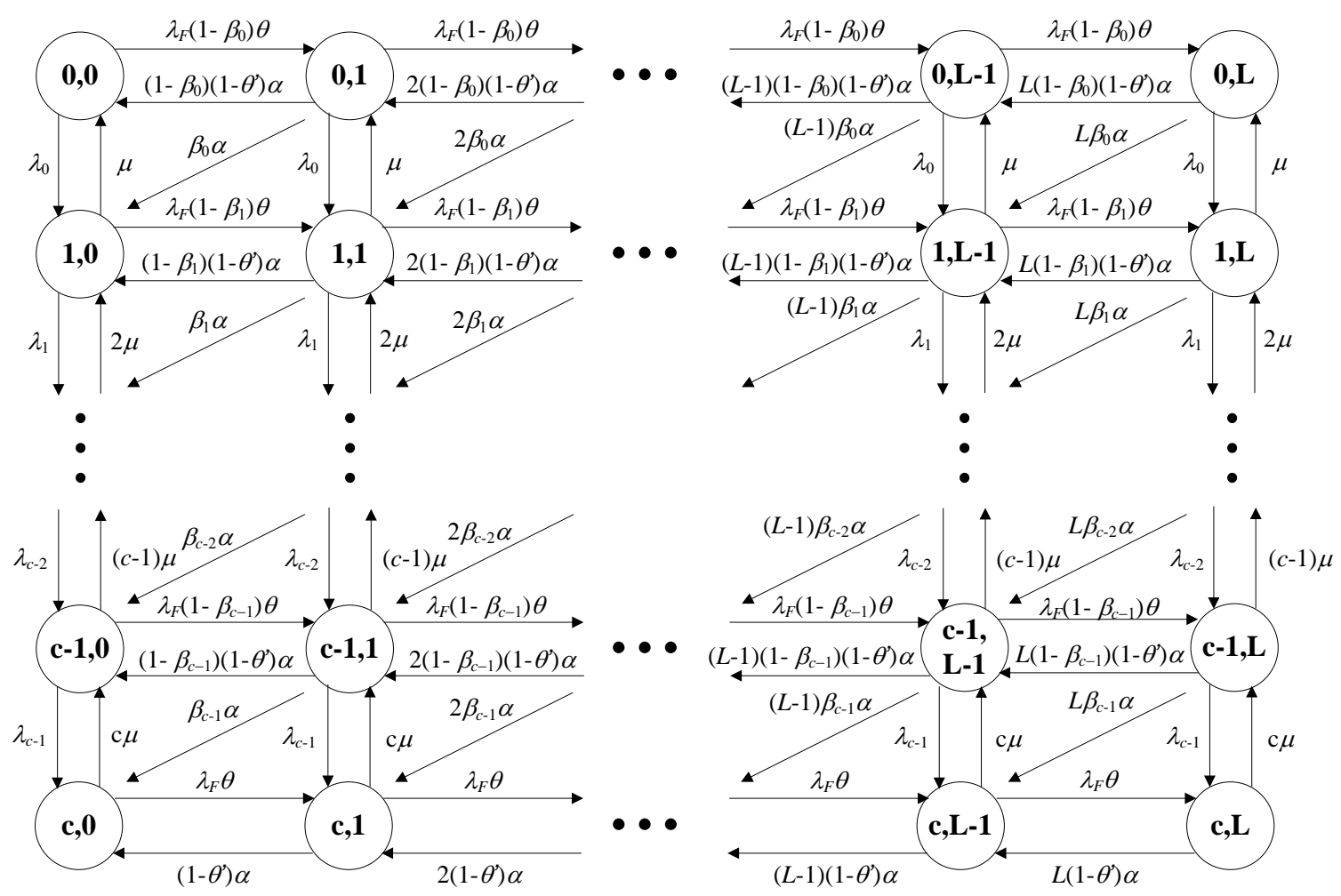

Figure 2. State transition diagram of the model

Let $\pi_{i, j}=\lim _{t \rightarrow \infty} P(I(t)=i, J(t)=j)$, where $\pi_{i, j}$ are the steady-state probabilities for the state $(i, j)$. When the chains $X=\{I(t), J(t) ; t \geq 0\}$ are represented through the state transition matrixes $A_{j}, B_{j}, C_{j}$ (with theirdimension $(c+1) \times(c+1)$ ) [9-13]:

- $A_{j}(i, k)$ are the state transition matrixes for the states from $(i, j)$ to $(k, j)(0 \leq i, k \leq$ $c, 0 \leq j \leq L)$. They express an successful call, which is a fresh or handover call, or a call having been completed and departing. $A_{j}$ has the entries $A_{j}(i, i-1)=i \mu(i=\overline{1, c})$ and $A_{j}(i, i+1)=\lambda_{i}=\beta_{i} \lambda_{F}+\lambda_{H}(i=\overline{0, c-1})$ and the rest 0 . 
International Journal of Computer Networks \& Communications (IJCNC) Vol.13, No.4, July 2021

$$
A_{j}=A=\left(\begin{array}{ccccccc}
0 & \lambda_{0} & 0 & \cdots & 0 & 0 & 0 \\
\mu & 0 & \lambda_{1} & \cdots & 0 & 0 & 0 \\
\vdots & \vdots & \vdots & \vdots & \vdots & \vdots & \vdots \\
0 & 0 & 0 & \cdots & (c-1) \mu & 0 & \lambda_{c-1} \\
0 & 0 & 0 & \cdots & 0 & c \mu & 0
\end{array}\right),(j \geq 0)
$$

- $B_{j}(i, k)$ are the state transition matrixes for the states from $(i, j)$ to $(k, j+1)(0 \leq i, k \leq$ $c, 0 \leq j \leq L-1)$. They express a fresh call first arriving the system and entering the orbit with the probability $\theta . B_{j}$ has the entries $B_{j}(i, i)=\left(1-\beta_{i}\right) \theta \lambda_{F}(i=\overline{0, c})$ (note $B_{j}(c, c)=\theta \lambda_{F}$ due to $\left.\beta_{c}=0\right)$ and the rest 0 .

$$
B_{j}=B=\left(\begin{array}{cccc}
\sigma_{0} & \cdots & 0 & 0 \\
0 & \cdots & 0 & 0 \\
\vdots & \vdots & \vdots & \vdots \\
0 & \cdots & \sigma_{c-1} & 0 \\
0 & \cdots & 0 & \sigma_{c}
\end{array}\right),(j \geq 0)
$$

Where $\sigma_{i}=\left(1-\beta_{i}\right) \theta \lambda_{F},(0 \leq i \leq c)$.

- $C_{j}(i, k)$ are the state transition matrixes for the states from $(i, j)$ to $(k, j-1)(0 \leq$ $i, k \leq c, 1 \leq j \leq L)$. They express a retrial call returning the system. Then it is served or permanently departs due to its impatience and all channels that are busy. $C_{j}$ has the entries $C_{j}(i, i+1)=\beta_{i} j \alpha(i=\overline{0, c-1})$ and $C_{j}(i, i)=\left(1-\beta_{i}\right)(1-$ $\left.\theta^{\prime}\right) j \alpha(i=\overline{0, c})$ (note $C_{j}(c, c)=\left(1-\theta^{\prime}\right) j \alpha$ due to $\beta_{c}=0$ ) and the rest 0 .

$$
C_{j}=\left(\begin{array}{cccccc}
\sigma_{0, j} & \beta_{0} j \alpha & 0 & \cdots & 0 & 0 \\
0 & \sigma_{1, j} & \beta_{1 j \alpha} & \cdots & 0 & 0 \\
\vdots & \vdots & \vdots & \vdots & \vdots & \vdots \\
0 & 0 & 0 & \cdots & \sigma_{c-1, j} & \beta_{c-1} j \alpha \\
0 & 0 & 0 & \cdots & 0 & \sigma_{c, j}
\end{array}\right),(j \geq 1)
$$

Where $\sigma_{i, j}=\left(1-\beta_{i}\right)\left(1-\theta^{\prime}\right) j \alpha(0 \leq i \leq c, 1 \leq j \leq L)$.

We have the generator matrix $Q$ :

$$
Q=\left(\begin{array}{ccccc}
Q_{1}^{(0)} & Q_{2}^{(0)} & \mathbf{0} & \mathbf{0} & \mathbf{0} \\
Q_{0}^{(1)} & Q_{1}^{(1)} & \ddots & \mathbf{0} & \mathbf{0} \\
\mathbf{0} & \ddots & \ddots & Q_{2}^{(L-2)} & \mathbf{0} \\
\mathbf{0} & \mathbf{0} & Q_{0}^{(L-1)} & Q_{1}^{(L-1)} & Q_{2}^{(L-1)} \\
\mathbf{0} & \mathbf{0} & \mathbf{0} & Q_{0}^{(L)} & Q_{1}^{(L)}
\end{array}\right)
$$


International Journal of Computer Networks \& Communications (IJCNC) Vol.13, No.4, July 2021

Where:

$$
\left\{\begin{array}{c}
Q_{1}^{(0)}=A-D^{A}-D^{B} \\
Q_{1}^{(j)}=A-D^{A}-D^{B}-D^{C_{j}}(1 \leq j \leq L-1) \\
Q_{L}^{(0)}=A-D^{A}-D^{C_{L}} \\
Q_{0}^{(j)}=C_{j}(1 \leq j \leq L) \\
Q_{2}^{(j)}=B(0 \leq j \leq L-1)
\end{array}\right.
$$

Where $D^{X}\left(X=A, B, C_{j}\right)$ are the diagonal matrixes with the entries on the main diagonal that are the sum of the entries on respective row of the matrix $X$. We have:

$$
v Q=\underbrace{(0,0, \ldots, 0)}_{(c+1) \times(c+1)}
$$

Where $v=\left(v_{0}, v_{1}, \ldots, v_{L}\right)$.

Let the probabilities $(i, j)$ are defined by $p_{i, j}=\lim _{t \rightarrow+\infty} P(I(t)=i, J(t)=j)$, and the level probabilities vectors $v_{j}=\left(p_{0, j}, p_{1, j}, \ldots, p_{c, j}\right)(1 \leq j \leq L)$. The balance equations of the system are:

$$
\begin{gathered}
v_{0} Q_{1}^{(0)}+v_{1} Q_{0}^{(1)}=\underbrace{(0,0, \ldots, 0)}_{c+1} \\
v_{j-1} Q_{2}^{(j-1)}+v_{j} Q_{1}^{(j)}+v_{j+1} Q_{1}^{(j+1)}=\underbrace{(0,0, \ldots, 0)}_{c+1}(1 \leq j \leq L-1) \\
v_{L-1} Q_{2}^{(L-1)}+v_{L} Q_{1}^{(L)}=\underbrace{(0,0, \ldots, 0)}_{c+1} \\
\sum_{j=0}^{L} v_{j} \times\left(\begin{array}{c}
1 \\
1 \\
\vdots \\
1
\end{array}\right)
\end{gathered}
$$

From (5) and (9), we calculate $v$ :

$$
v=e(Q+E)^{-1}
$$

Where $e$ is the row vector $(1 \times(c+1))$ and $E$ is the matrix $((c+1) \times(c+1))$ all of whose elements are the constants 1 .

\subsection{The Performance Measures of the System}

The probability of the fresh calls $P B_{F}$ firstly arrives the system and find all busy channels at the time or is rejected with the probability $\left(1-\beta_{i}\right)$. 
International Journal of Computer Networks \& Communications (IJCNC) Vol.13, No.4, July 2021

$$
P B_{F}=\sum_{j=0}^{L} \sum_{i=0}^{c} p_{i, j}\left(1-\beta_{i}\right)
$$

The probability of the retrial calls $P B_{F, \text { retrial }}$ then arrives the system from the orbit and find all busy channels at the time is rejected with the probability $\left(1-\theta^{\prime}\right)$.

$$
P B_{F, \text { retrial }}=\frac{\left(1-\theta^{\prime}\right) \alpha}{\theta \lambda_{F}} \sum_{j=1}^{L} \sum_{i=0}^{c} j p_{i, j}
$$

The probability of the handover calls $P B_{F, \text { retrial }}$ clearly arrives the system and find all busy channels at the time.

$$
P B_{H}=\sum_{j=0}^{L} p_{c, j}
$$

\section{RESUltS}

The efficiency of the performance as the change of the parameters of the system is firstly considered with the fresh and handover calls. When the blocks occur, the fresh calls reattempt to connect in the intervals of the stochastic distribution. We assume the base station of the cell that can process $c$ the connection simultaneously. Table 1 enumerates the parameters to analyze results. The Mathematica program of Wolfram Research denoted [14] is a power tool to compute and simulate for network models and is utilized in the proposed model of the paper.

Table 1. The parameters of the model.

\begin{tabular}{|l|l|l|}
\hline The parameters & Values & Describe the parameters \\
\hline$c$ & 10 & Number of the channels in the cell \\
\hline$g$ & 1 to 7 & Number of the accepted channels \\
\hline$p$ & 0.1 to 1 & Probability that a fresh call is served \\
\hline$L$ & 1 to 10 & Number of the customers in the orbit \\
\hline$\lambda_{H}$ & 0.002 to 2 & Arrival rate for the handover calls \\
\hline$\lambda_{F}$ & 0.018 to 0.2 & Arrival rate for the fresh calls \\
\hline$\mu$ & 0.2 & Service rate \\
\hline$\alpha$ & 0.4 & Retrial rate \\
\hline$\theta$ & 0.1 to 1 & Probability that a fresh call first enters the orbit \\
\hline$\theta^{\prime}$ & 0.1 to 1 & Retrial probability that a fresh call re-enters the orbit \\
\hline
\end{tabular}

\subsection{Analysing and Comparing the Blocking Probabilities with Values $\rho$}

First, we compare the blocking probabilities $P B\left(P B_{F}, P B_{F, \text { retrial }}\right.$ or $\left.P B_{R}, P B_{H}\right)$ according to a variety of the fractional guard channel policies with the fluctuations in values $\rho$. It can be seen in Figure 3, the value $\rho$, the traffic load, is definited by the expression $\rho=\lambda / c \mu$. We have $g=7$, $p=0.5, r=2.3$ (for the LAFGC policy), $L=8, \theta=0.5, \theta^{\prime}=0.5, \lambda_{F}=2 \lambda_{H}, \rho$ ranging from 0.3 to 1.9 . We find that the blocking probabilities for the LFGC and LAFGC policies are better than the blocking probabilities for the UFGC and QUFGC policies. 
International Journal of Computer Networks \& Communications (IJCNC) Vol.13, No.4, July 2021

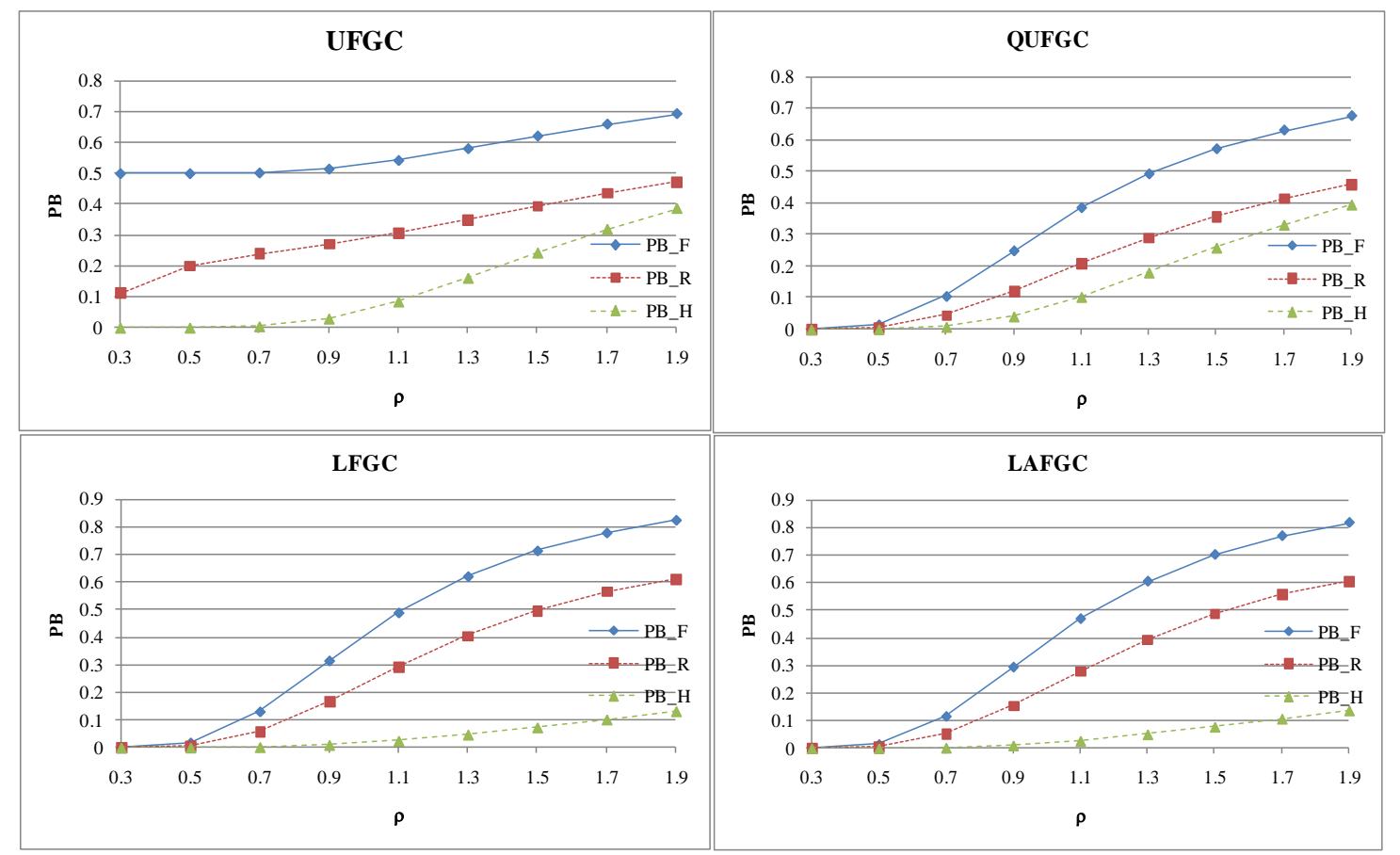

Figure 3. The blocking probability of $P B_{F}, P B_{F, \text { retrial }}$ and $P B_{H}$ by $\rho$.

This may partly explain that the UFGC and QUFGC policies permit the fresh calls with $\beta_{i}=1$ or $\beta_{i}=p(0 \leq i<c)$. As a result, the fresh calls are easier to enter the system, and the handover calls are significantly restricted. In the meanwhile, the LFGC and LAFGC policies control the fresh calls with $\beta_{i}=0(0<L \leq i<c, L$ : a particular parameter $)$ at some of the last states with $i \geq L$.

\subsection{Analysing and comparing the blocking probabilities with valuesg}

With $\rho=0.7, p=0.5, L=8, \theta=0.5, \theta^{\prime}=0.5, \lambda_{F}=2 \lambda_{H}, g$ ranging from 1 to 7 , we obtain the results as presented in Figure 4.

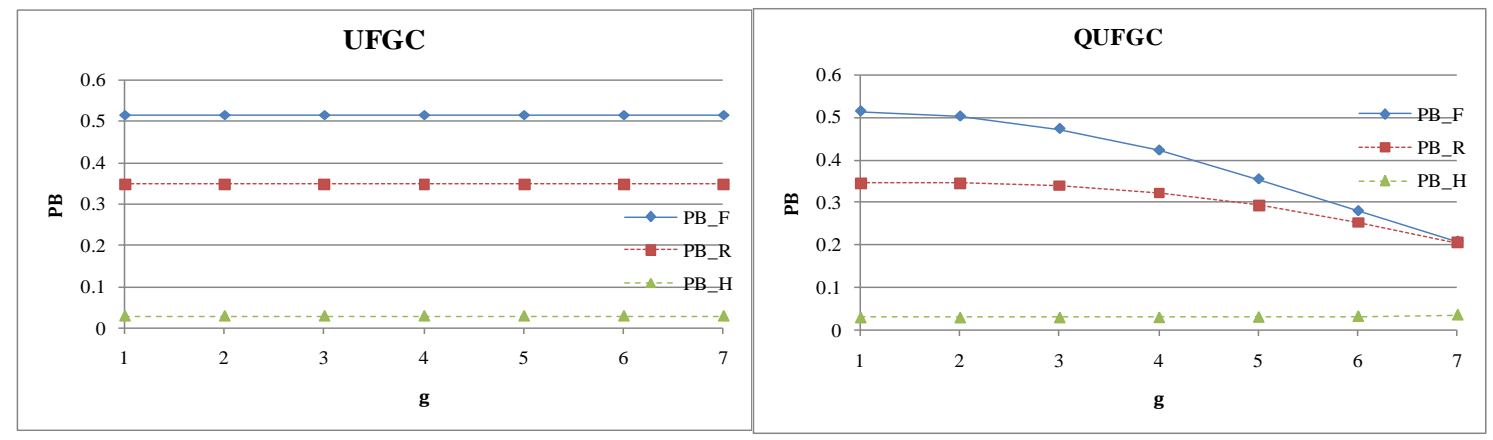


International Journal of Computer Networks \& Communications (IJCNC) Vol.13, No.4, July 2021
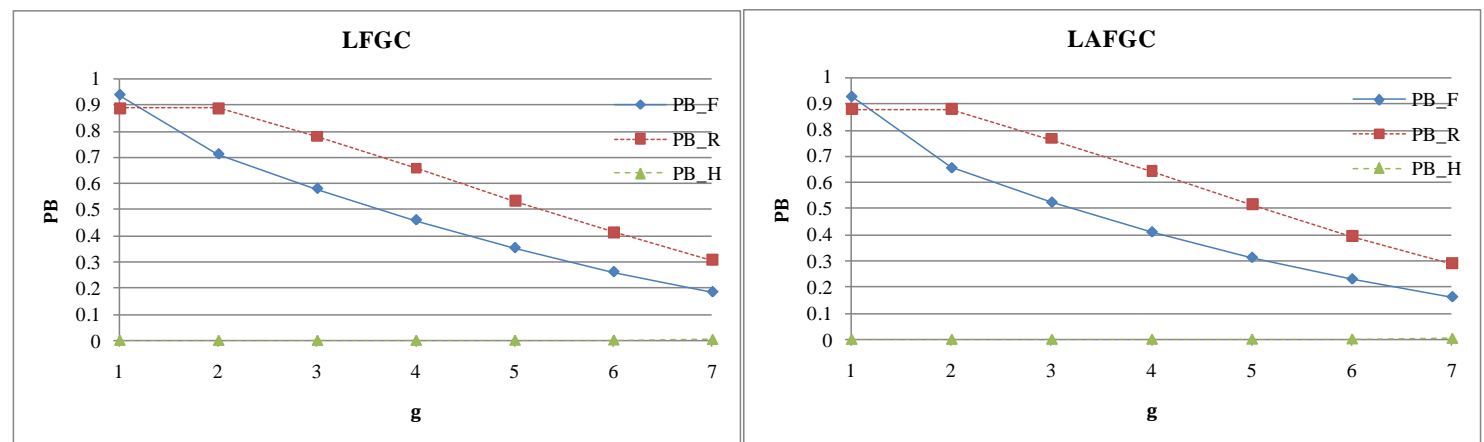

Figure 4. The blocking probability of $P B_{F}, P B_{F, \text { retrial }}$, and $P B_{H}$ by $g$.

When we change values $g$, the probabilities $P B_{F}$ and $P B_{F, \text { retrial }}$ for the QUFGC policy decrease inversely proportional to values $g$, and the probabilities $P B_{H}$ for the QUFGC, UFGC, LFGC and LAFGC policies remain steady and thus we need to choose an appropriate value $g$ for the low blocking probabilities $P B_{H}$. The UFGC policy has the probabilities $P B_{F}$ and $P B_{F, \text { retrial }}$ which maintain stable due to $\beta_{i}=p(0 \leq i<c)$. Similar to the QUFGC policy, the probabilities $P B_{F}$ and $P B_{F, \text { retrial }}$ for the LFGC and LAFGC policies significantly decline because the numbers of the accepted channels for the fresh and retrial calls gradually increase.

\subsection{Analysing and Comparing the Blocking Probabilities with Values $L$}

With $\rho=0.7, g=7, p=0.5, r=2.3$ (for the LAFGC policy), $\theta=0.5, \theta^{\prime}=0.5, \lambda_{F}=2 \lambda_{H}, L$ ranging from 1 to 8 , we obtain the results presented in Figure 5.
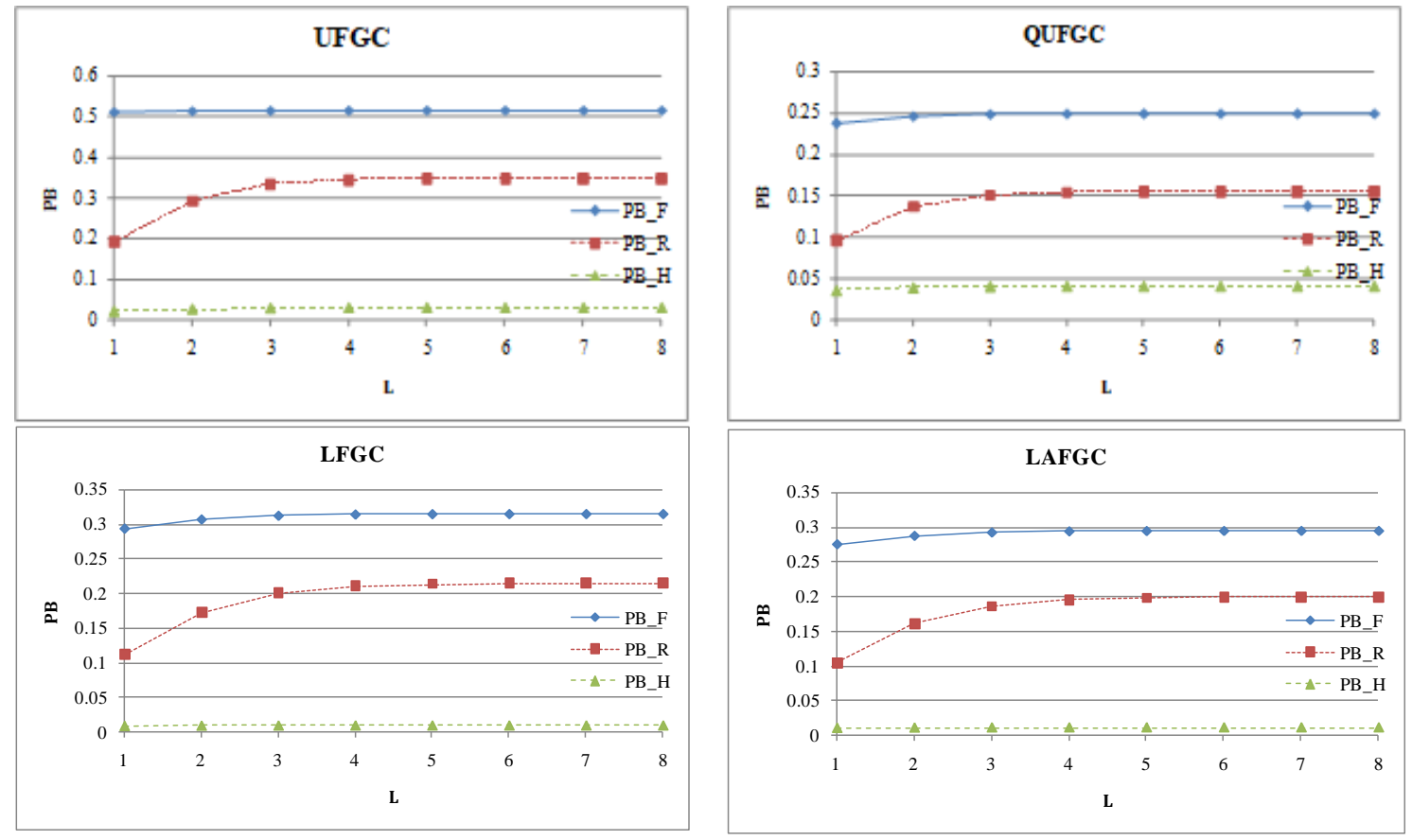

Figure 5. The blocking probability of $P B_{F}, P B_{F, \text { retrial }}$, and $P B_{H}$ by $L$.

The blocking probabilities $P B_{F}$ and $P B_{F, \text { retrial }}$ are influenced by the number of the maximum customers in the orbit $L$ when $L<4$ as Figure 5. When $L \geq 4$ these probalities are almost 
International Journal of Computer Networks \& Communications (IJCNC) Vol.13, No.4, July 2021

steady. While the blocking probabilities $P B_{H}$ are not affected regardless of the value $L$. This can be explained that the number of the servers is fixed, the more the customers in the orbit are, the more blocked the system is. For this reason, we can approximate to the infinite model with $L \rightarrow$ $+\infty$.

\subsection{Analysing and comparing the blocking probabilities with values $p$}

With $\rho=0.7, g=7, p=0.5, L=8, r=2.3$ (for the LAFGC policy), $\theta=0.5, \theta^{\prime}=0.5, \lambda_{F}=$ $2 \lambda_{H}, p$ ranging from 0.1 to 1 , we obtain the results as listed in Figure 6.

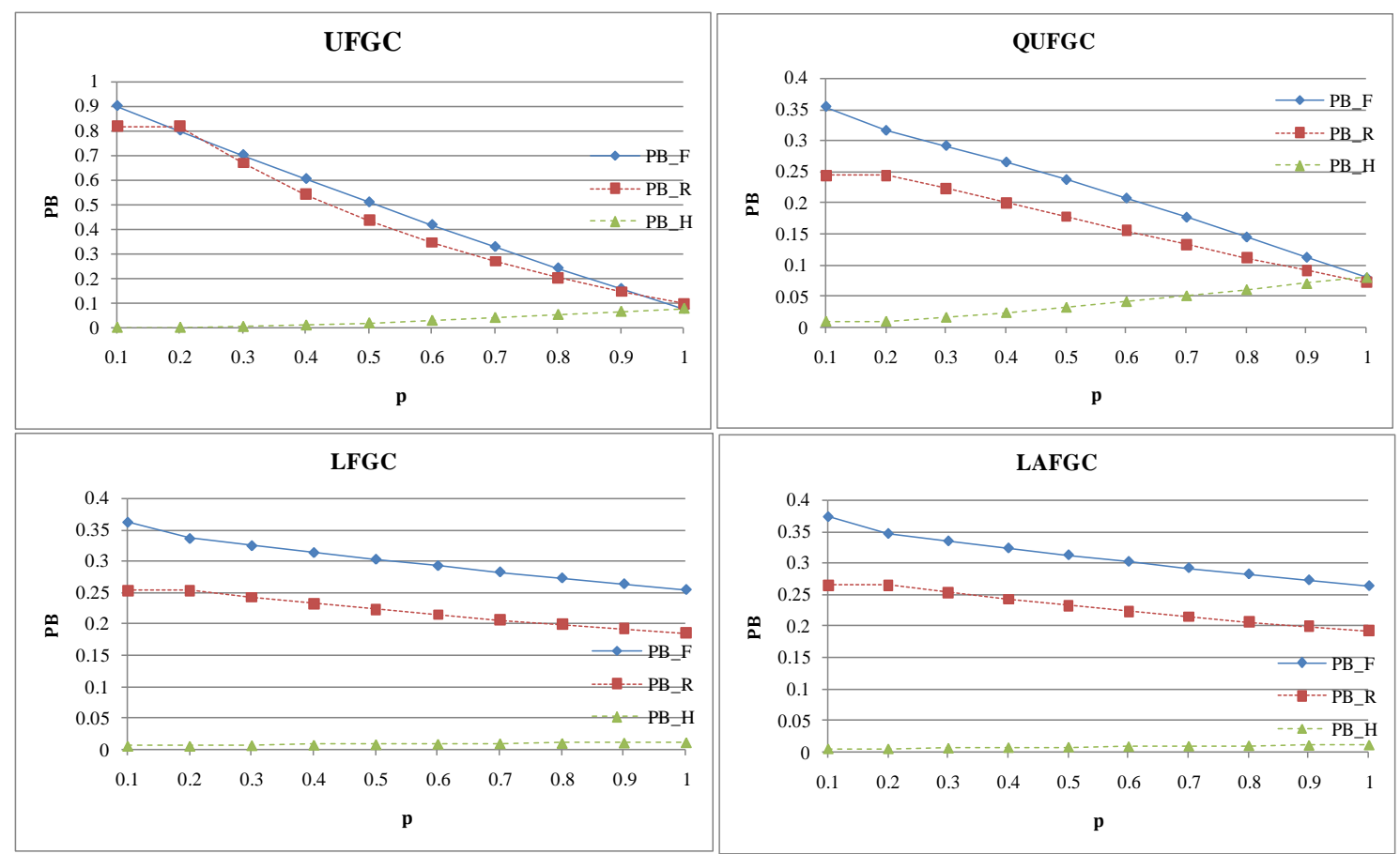

Figure 6. The blocking probability of $P B_{F}, P B_{F, \text { retrial }}$, and $P B_{H}$ by $p$.

We found/find that the accepted probabilities of the fresh calls $p$ influence on how the congestions of the fresh and retrial calls are. In these analyses, the blocking probabilities of the fresh calls for the UFGC and QUFGC policies are better than the ones for the LFGC and LAFGC policies due to the fact that the the probabilities $\beta_{i}(0 \leq i \leq c)$ for the LFGC and LAFGC policies have more the values $p$ than the ones for the UFGC and QUFGC policies. As a result, the fresh and retrial calls ease to enters the system for the LFGC and LAFGC policies.

\subsection{Analysing and comparing the blocking probabilities with values $\lambda_{H} / \lambda_{F}$}

With $\rho=0.7, g=7, L=8, p=0.5, \theta=0.5, \theta^{\prime}=0.5, \lambda_{H} / \lambda_{F}$ ranging from $1 / 5$ to 5 , we obtain the results as shown in Figure 7. 
International Journal of Computer Networks \& Communications (IJCNC) Vol.13, No.4, July 2021

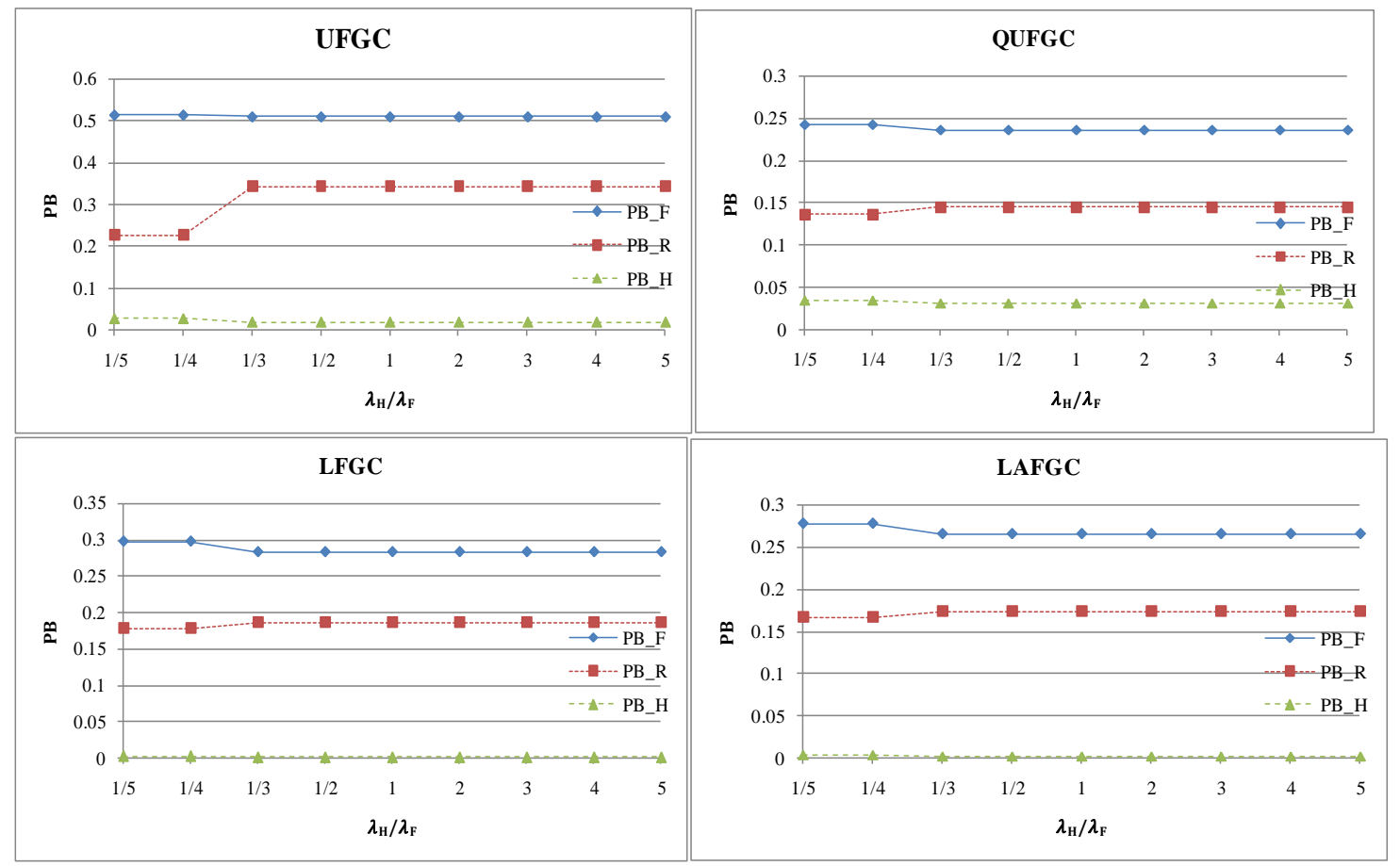

Figure 7. The blocking probability of $P B_{F}, P B_{F, \text { retrial }}$, and $P B_{H}$ by $\lambda_{H} / \lambda_{F}$.

We find that if the ratio $\lambda_{H} / \lambda_{F}<1 / 3$, the probabilities $P B_{F}, P B_{F, r}$ and $P B_{H}$ are changed, but if the ratio $\lambda_{H} / \lambda_{F} \geq 1 / 3$, the probabilities $P B_{F}, P B_{F, r}$ and $P B_{H}$ remain almost unchanged. Therefore, we choose the ratio $\lambda_{H} / \lambda_{F} \geq 1 / 3$ to maintain the stability of the system.

\subsection{Analysing and comparing the blocking probabilities with values $\theta$}

With $\rho=0.7, g=7, L=8, p=0.5, r=2.3$ (for the LAFGC policy), $\theta^{\prime}=0.5, \lambda_{F}=2 \lambda_{H}, \theta$ ranging from 0.1 to 1 , we gain the results as presented in Figure 8 .
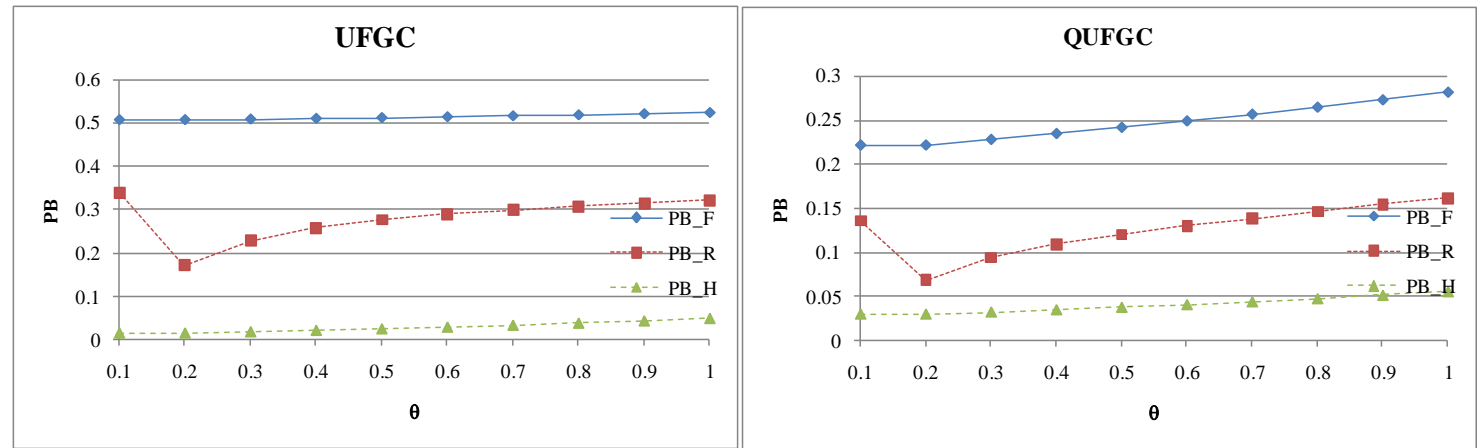
International Journal of Computer Networks \& Communications (IJCNC) Vol.13, No.4, July 2021
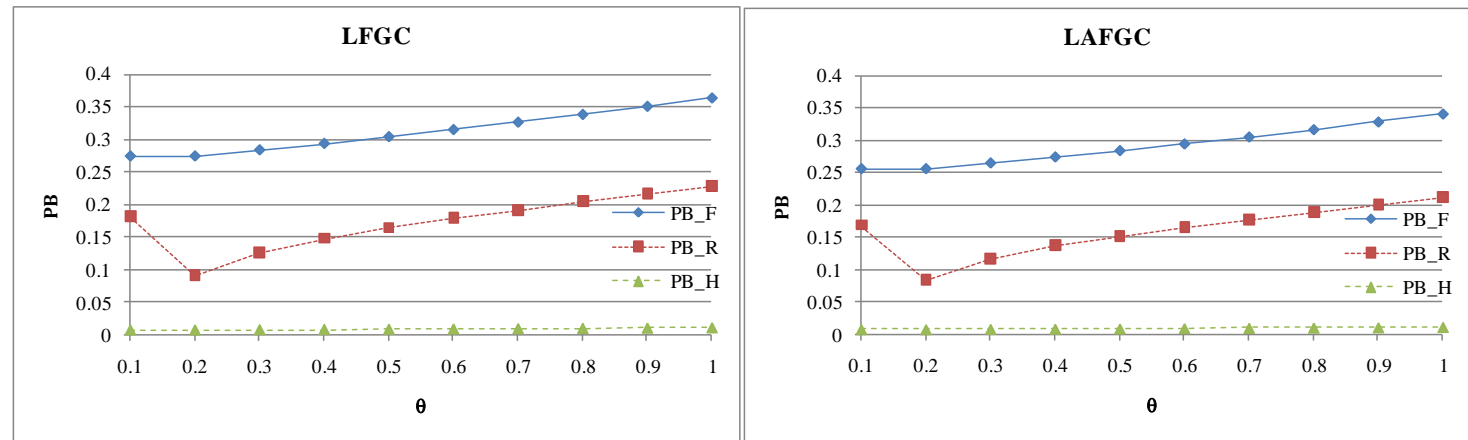

Figure 8 . The blocking probability of $P B_{F}, P B_{F, \text { retrial }}$, and $P B_{H}$ by $\theta$.

The parameter $\theta$ gives the influence on the blocking probabilities of the retrial calls rather than the ones of the fresh calls. This can be explained that the probabilities that a fresh call first enters the orbit increase resulting in a numerous number of the customers in the the orbit, and the blocking probabilities of the retrial calls will raise. In the meantime, the probabilities of the handover calls remain almost unchanged. In these analyses, we find that the parameters $\in$ $(0.2-\varepsilon, 0.2+\varepsilon)$, with $\varepsilon \geq 0$ is a arbitrarily small real. The system performances are optimized by the given parameters.

\subsection{Analysing and comparing the blocking probabilities with values $\theta^{\prime}$}

With $\rho=0.7, g=7, L=8, p=0.5, r=2.3$ (for the LAFGC policy), $\theta=0.5, \lambda_{F}=2 \lambda_{H}, \theta^{\prime}$ ranging from 0.1 to 1 , we obtain the results as presented Figure 9.

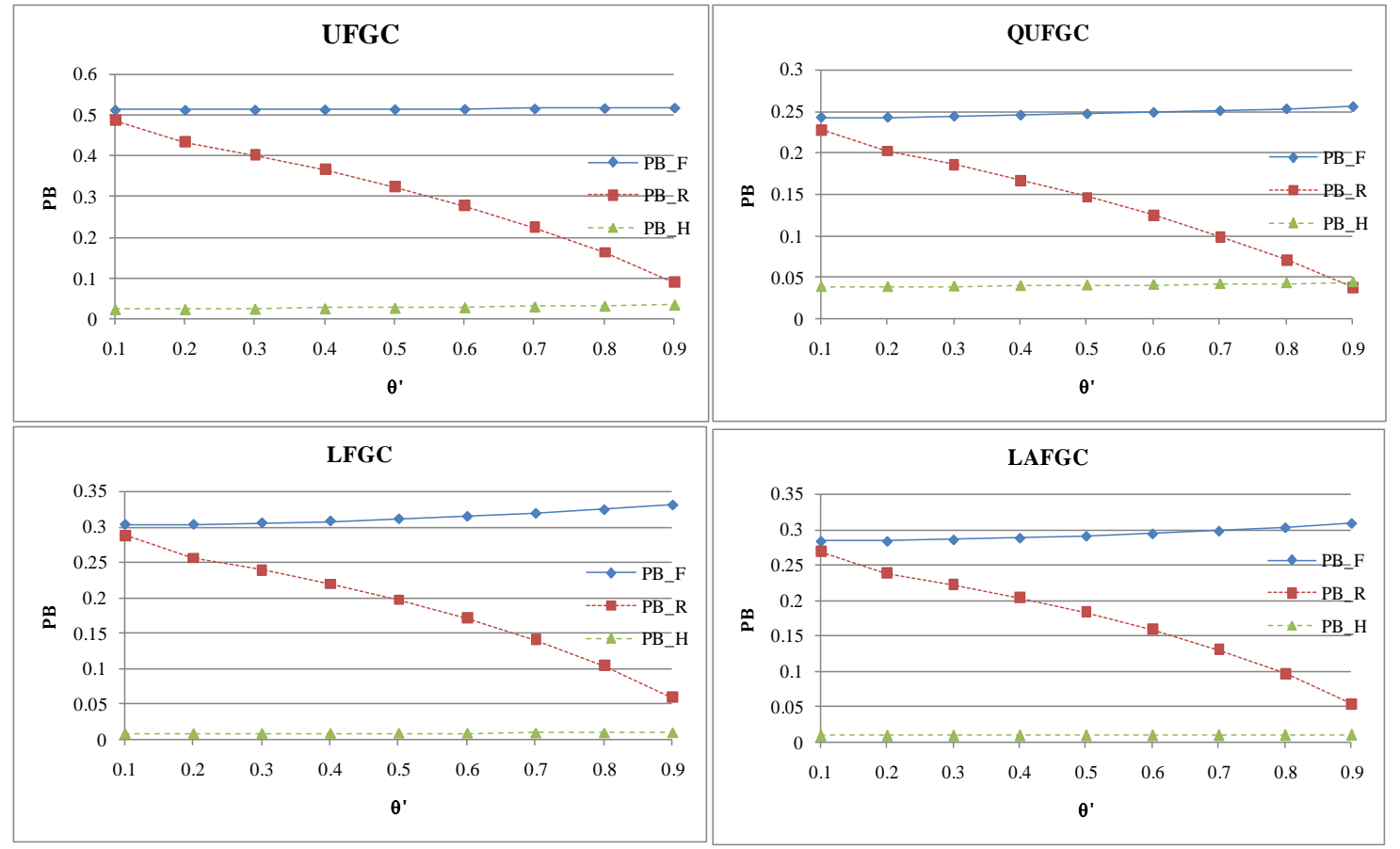

Figure 9. The blocking probability of $P B_{F}, P B_{F, \text { retrial }}$, and $P B_{H}$ by $\theta^{\prime}$.

When taking into account the increase in the retrial probabilities $\theta^{\prime}\left(0.1 \leq \theta^{\prime} \leq 0.9\right)$, the blocking probabilities $P B_{F, r}$ are declined. Then the retrial calls re-enter the orbit to attempt the 
International Journal of Computer Networks \& Communications (IJCNC) Vol.13, No.4, July 2021

next occasions. On the contrary, the blocking probabilities $P B_{F}$ and $P B_{H}$ are slightly affected. As the results ofinFigure 9, when the parameter $\theta^{\prime}$ tends to the asymptotic value of 1 , the blocking probabilities $P B_{F, r}$ almost optimize the certain performances.

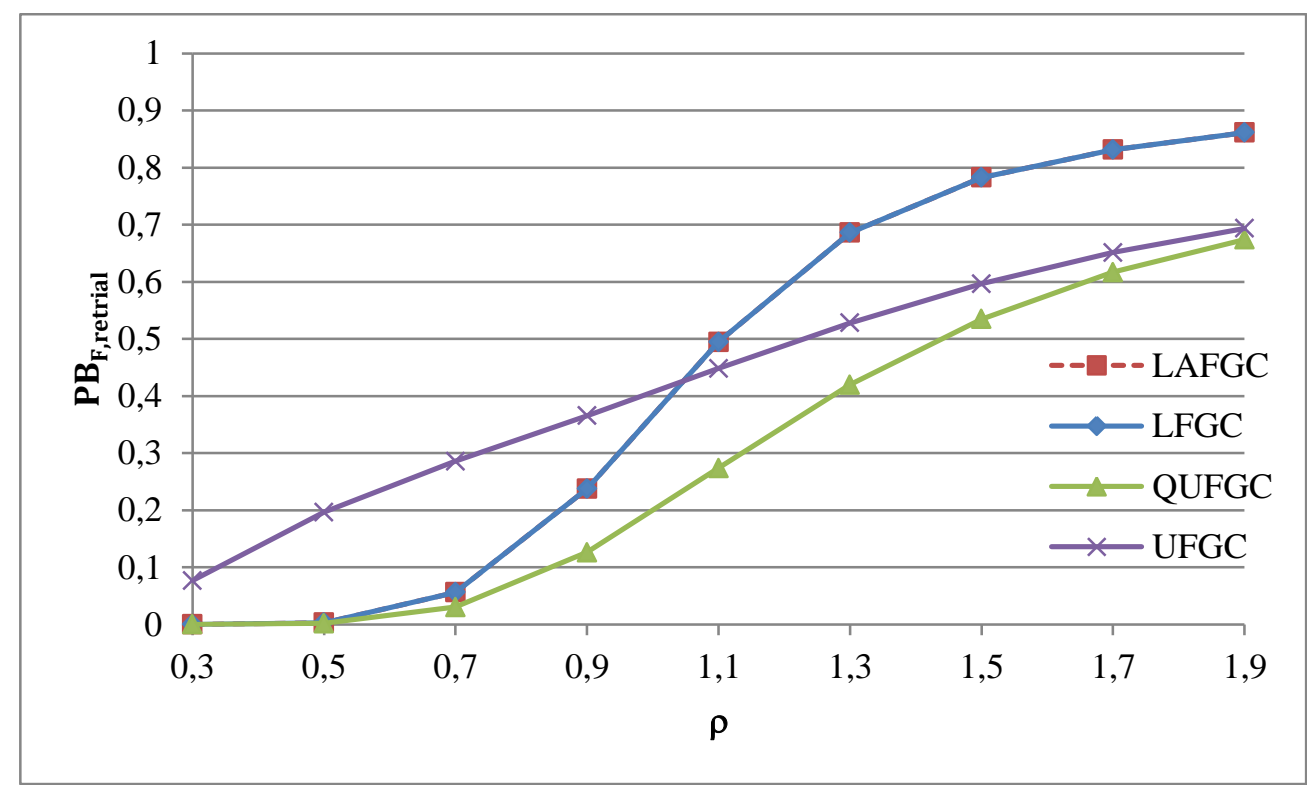

(a)

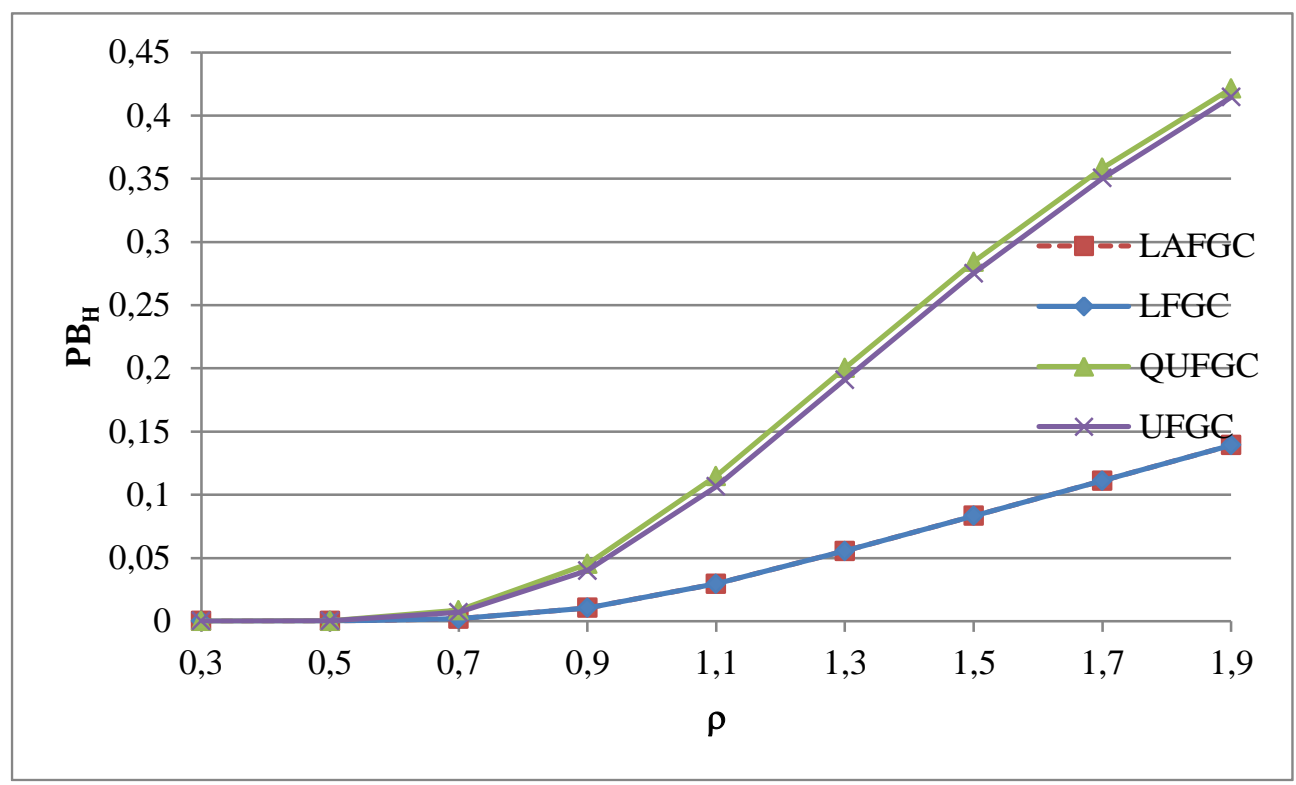

(b)

Figure 10. The blocking probability of $P B_{F, \text { retrial }}$ (a) and $P B_{H}$ (b) among LAFGC, LFGC, QUFGC and UFGC

We find that the blocking probabilities $P B_{F, \text { retrial }}$ and $P B_{H}$ of LAFGC and LFGC are almost equivalent (Figure 10). Also, the blocking probabilities $P B_{H}$ of QUFGC and UFGC are approximately equal, yet the blocking probabilities $P B_{F, r}$ of QUFGC are better than those of UFGC. This is explained that the accepted probabilities $\beta_{i}$ of QUFGC are 1 at some initial states. 
International Journal of Computer Networks \& Communications (IJCNC) Vol.13, No.4, July 2021

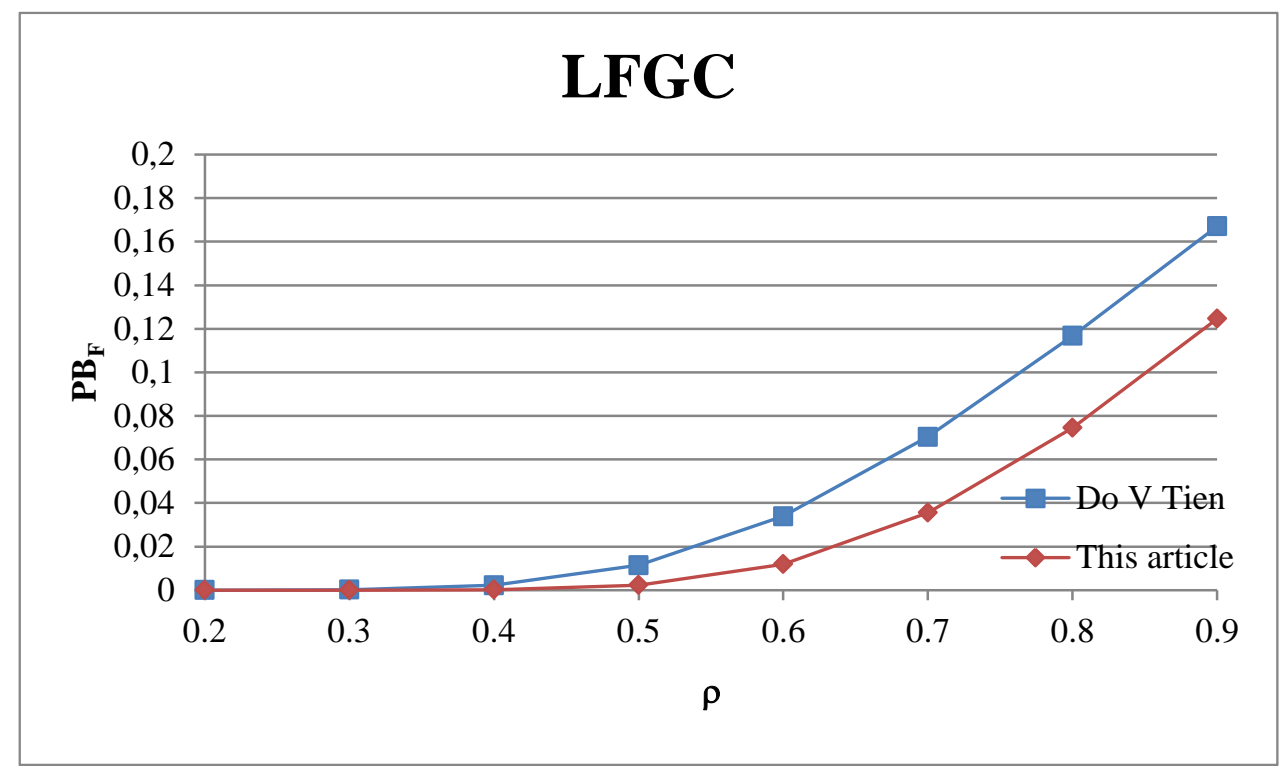

Figure 11. The blocking probability of $P B_{F}$ between in [7] and the article.

According to figures extracted from [7] (of Do Van Tien) with $L=c-1, \beta=0, c=15, \mu=$ $\frac{1}{120}, \alpha=20 \mu, \lambda_{F}=24 \lambda_{H}$, we ascertain that the probabilties of $P B_{F}$ in the article are superior to those of [7]. The disparity is that the further value $\theta^{\prime}$ can be varied, depending on users's adjustments and playing a signal role in reducing the possibilities of obstructing the fresh calls.

\section{Conclusions}

We have introduced the retrial queueing model in the cellular mobile network using the fractional guard channel policy with impatient customers according to the probabilities $\theta$ and $\theta^{\prime}$. The advantage of the model is the significant decrease of the blocking probabilities for the fresh and retrial calls as well as the support of the network operation to maintain the stability of QoS under the influences on the network traffic and additional factors. The generality in the model is weighed by choosing the retrial queueing model in combination with the fractional guard channel policies. The analysis results are simple and guarantee the low blocking probabilities of the handover calls for the fractional guard channel policy in compassion to the blocking probabilities of the guard channel policy. In addition, the retrial queueing model with the fractional guard channel policy in the cellular mobile network can be used for the criteria of the different network traffics to measure the performances. The corollaries elucidate that the model is appropriate and precise. Besides, the research considers a variety of the fractional guard channel policies, including the UFGC, QUFGC, LFGC, and LAFGC policies, to fall the blocking probabilities of the fresh calls, but they still guarantee the handover calls protected with the call admission control.

\section{CONFLICTS OF INTEREST}

The authors declare no conflict of interest. 
International Journal of Computer Networks \& Communications (IJCNC) Vol.13, No.4, July 2021

\section{REFERENCES}

[1] Mandles M. and Tutschku K., (1996). Efficient Call Handling Procedures in Cellular Mobile Networks.Forschungsbericht, Preprint-Reihe 144, Institut fur Informatik, Universitat Wurzburg, Germany.

[2] Tran-Gia P. and Mandles M., (1997). Modeling of Customer Retrial Phenomenon in Cellular Mobile Networks.IEEE Journal on Selected Areas in Communications, 15(8), pp. 1406-1414.

[3] Do T. V. (2010). A new computational algorithm for retrial queues to cellular mobile systems with guard channels. Computers \& Industrial Engineering, 59(4), pp. 865-872.

[4] Linda A. C. and Ani C. I. (2014). A Reservation and Retrial Queue Analysis for Cellular Networks.International Journal of Engineering Research \& Technology (IJERT), ISSN: 2278-0181, Vol. 3 Issue 2, February - 2014

[5] Bhargava A., Bhagat A. and Singh J., (2016). Modelling and Performance Analysis of Cellular Mobile Networks.Indian Journal of Science and Technology, Vol 9 (48), December 2016.

[6] Vazquez-Avila J., Cruz-Pérez F.A. and Ortigoza-Guerrero L., (2006). Performance Analysis of Fractional Guard Channel Policies in Mobile Cellular Networks.IEEE Transactions on Wireless Communications, 5 (2006), pp. 301-305.

[7] Do T. V. (2011). Solution for A Retrial Queueing Problem in Cellular Networks with the Fractional Guard Channel Policy.Mathematical and Computer Modelling, 53(11-12), pp. 2058-2065.

[8] Ramjee R., Towsley D. and Nagarajan R., (1997). On Optimal Call Admission Control in Cellular Networks.Wireless Network, 3(1), March 1997.

[9] Neuts M.F. (1981). Matrix Geometric Solutions in Stochastic Model, Johns Hopkins University Press, Baltimore.

[10] Tien Van Do, Ram Chakka, An efficient method to compute the rate matrix for retrial queues with large number of servers, Applied Mathematics Letters 23, (2010) 638-643.

[11] Tien, D.V. (2013). An Enhanced Algorithm to Solve Multiserver Retrial Queueing Systems with Impatient Customer. Computers \& Industrial Engineering, Vol 65, No 4, pp. 719-728.

[12] Kulshrestha R, Agarwal A, Shruti (2019). An adaptive fractional guard channel based CAC scheme for heterogeneous traffic in wireless cellular networks. In: Proceedings of IEEE 6th international conference in computing for sustainable global development, Delhi, India, pp 1066-1070.

[13] Do T.V. (2010). An Efficient Computation Algorithm for A Multiserver Feedback retrial Queue With A Large Queueing Capacity, Applied Mathematical Modelling, 34(8), pp. 2272-2278.

[14] https://www.wolfram.com/mathematica/ 


\section{AUTHORS}

Dang Thanh Chuong obtained his doctorate in Mathematical Foundation for Computers and Computing Systems in 2014 from the Institute of Information Technology, Vietnam Academy of Science and Technology (VAST). He has published over 20 research papers. His research interests are in the fields of all-optical networks with emphasis on packet/burst-based switching, Contention Resolution, and Quality of Service; Queueing Theory and Retrial Queue. Email: dtchuong@hueuni.edu.vn.

Hoa Ly Cuong procuring MSc in Computer Science in 2017 from the Hue University of Science, Hue University. The areas he has engaged in comprise Queueing Theory and Wireless Networks.

Email: hlcuong90@gmail.com.

Pham TrungDuc In 2010, he graduated with a Bachelor of Information Technology from University of Sciences, Hue University. In 2012, he received a Master's degree in Computer Science from Hue University of Sciences. Currently, he is a PhD student at Hue University of Sciences, Hue University (from December 2016 to the present).

Research fields: OBS network, QoS differentiation, scheduling admission control, QoS improvement, QoS provisioning. Email: phamtrungduc@hueuni.edu.vn
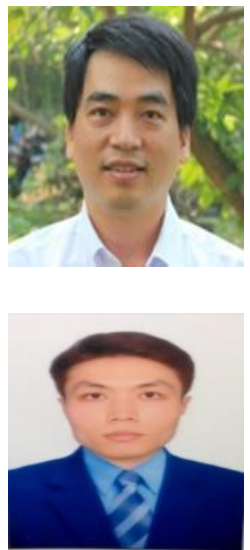

Duong Duc Hung is a Technical Editor at the HU Journal of Science, Hue University, Vietnam. His main research topics are Computer Networks and Communications; Text Mining.

Email: ddhung@hueuni.edu.vn

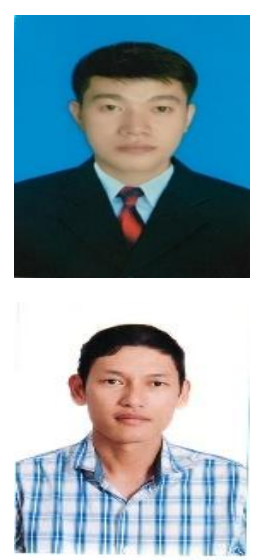

